\title{
Noise Reduction Performance of Various Signals for Impulse Response Measurement
}

\author{
YUTAKA KANEDA, AES Member \\ (kaneda@c.dendai.ac.jp) \\ Department of Information and Communication Engineering, Faculty of Engineering, Tokyo Denki University, \\ 5 Senju-Asahi-cho, Adachi-ku, Tokyo 120-8551, Japan
}

\begin{abstract}
In the measurement of impulse response, ambient noise is included leading to a decrease in measurement quality. To solve this problem the use of several measurement signals (or excitation signals) has been proposed. However, the relationship between measurement signals and the noise reduction performance $(N R P)$ has not been quantitatively examined thus far. In this study the $N R P$ characteristics of different measurement signals were theoretically examined to derive equations that can determine $N R P$ from the spectra of the measurement signal and noise. From the theoretical and experimental examinations the following results were obtained. The NRP for white signals and noise-whitening signals is actually the same. Only the minimum noise $(\mathrm{MN})$ signal that minimizes the noise component showed a significant improvement in $N R P$. A pink spectrum measurement signal showed good $N R P$ in the presence of $1 / \mathrm{k}^{2}$ spectrum noise, where $\mathrm{k}$ is the discrete frequency number, but worse performance with other types of noise. This supports the conclusion that using the MN signal, which has a power spectrum that is the square root of the power spectrum of the noise, is the best method of reducing the effect of noise on the measured impulse response.
\end{abstract}

\section{INTRODUCTION}

Impulse response, as well as its Fourier transform, i.e., frequency response, is a basic characteristic of acoustic systems. Various characteristics related to architectural acoustics including the reverberation time and speech transmission index (STI) are calculated using the impulse response [1]. In addition, simulations in the studies of sound field reproduction and architectural acoustics have been carried out using the measured impulse response [2]. The impulse response is also used directly or indirectly for sound field control and active noise control [3]. Thus, the impulse response is an essential characteristic of acoustic systems. In an actual environment, however, the measurement quality of the impulse response deteriorates because acoustic ambient noise and electrical noise (hereafter collectively referred to as noise) are included in the measurement results.

To address this problem, the use of various measurement signals for the impulse response (or excitation signals; hereafter, measurement signals) has been proposed. A linearly swept sine (SS) signal, also known as a time-stretched pulse (TSP) signal [4]-[6], and a maximum length sequence (MLS) signal [7]-[9] are known as basic measurement signals. Compared with the impulse signal, the energies of these measurement signals are high, enabling them to reduce the noise component included in the measurement results.
However, the linearly SS and MLS signals are white signals that have a constant power over the entire frequency range. Hence, when a room noise that has a high energy in a low-frequency range is added, the signal-to-noise (SN) ratio in the low-frequency range decreases. In contrast, a logarithmic swept sine (log-SS) signal, also known as an exponential sine sweep (ESS) signal [10]-[12], whose logarithmic frequency proportionally increases with time, has a high energy in the low-frequency range. Therefore, a high noise reduction performance can be obtained in the lowfrequency range.

Air conditioners are major noise sources that produce noise with a high energy in the low-frequency range. However, the shape of the spectrum depends on the type of air conditioner and the room environment, resulting in variations in the noise reduction performance. In addition, when there is a machinery noise source in the neighborhood, there may be noises with high energies in the medium-to-highfrequency range and such noises cannot be fully reduced using the log-SS signal.

To solve this problem, noise-adaptive measurement signals, which are synthesized by considering the preliminarily examined noise spectra, have been proposed. Weinzierl et al. used a measurement signal with a power spectrum identical to that of the measured noise [13]. Here, the effect of the noise biased to particular frequencies can be reduced because the noise component included in the measurement 
(a)

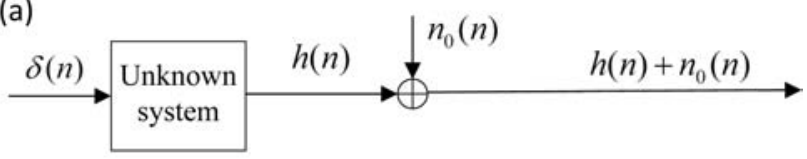

(b)

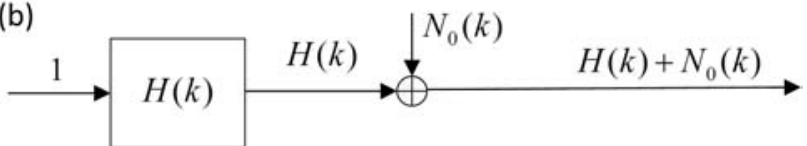

(c)

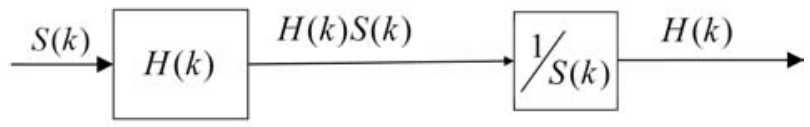

(d)

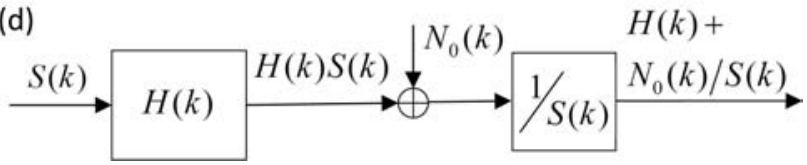

Fig. 1 Impulse response measurement in a noisy environment. (a) Measurement result obtained using impulse signal $\delta(n)$ and noise. (b) Frequency domain representation of (a). (c) Frequency domain representation of impulse response measurement using measurement signal S(k) under noise-free condition. (d) Frequency domain representation when noise is added to (c)

results is whitened by this signal. In this study we refer to this signal as a noise-whitening (NW) signal.

Moriya and Kaneda proposed a signal that can minimize the noise component included in measurement results (referred to as the minimum noise (MN) signal) [14], [15]. The MN signal has a power spectrum that is the square root of the power spectrum of the noise.

As explained above, various measurement signals for reducing the effect of noise on measurement results have been proposed thus far. However, the noise reduction performance of each measurement signal has not been sufficiently or quantitatively examined. In this study we examined the noise reduction performance of each measurement signal theoretically and experimentally.

In Sec. 1, the principles of the impulse response measurement and the noise component included in the measurement result are explained. In Sec. 2, the results of a theoretical examination of the noise reduction performance (NRP) characteristics of measurement signals are discussed. In Sec. 3, the results of a simulation of an impulse response measurement carried out to verify the results discussed in Sec. 2 are given and discussed. Finally, the study is concluded in Sec. 4.

\section{MEASUREMENT OF IMPULSE RESPONSE IN NOISY ENVIRONMENT}

In this study discrete-time signals are considered. As the frequency domain representation of the signal, the L-point discrete Fourier transform (DFT) is considered.

Fig. 1(a) shows a model diagram for an impulse response measurement in a noisy environment. When a discrete-time impulse signal $\delta(n)$ is input to an unknown system, the impulse response $h(n)$ is the system output with the noise $n_{0}(n)$ added. Here, $n$ is the discrete time and the added noise is assumed to be a stationary noise.

Fig. 1(b) shows the DFT frequency domain representation of Fig. 1(a). $H(k)$ is obtained by applying the L-point DFT to $h(n)$ and represents the frequency responses of the unknown system. Here, $k$ is the discrete frequency number and $N_{0}(k)$ is obtained by applying the L-point DFT to $n_{0}(n)$. The measurement result is $H(k)+N_{0}(k)$.

The SN ratio of the measurement result obtained using the impulse signal $\delta(n)$ is small because of its small energy. Therefore, measurement signals with high energies are generally used. Fig. 1(c) shows a model diagram for the measurement of impulse response using a measurement signal in an environment without noise. $S(k)$ is the DFT spectrum of the measurement signal.

When $S(k)$ is input to the unknown system, the output is $H(k) S(k)$. To obtain a product in the DFT frequency domain circular convolution should be carried out in the time domain. Thus, two periods of a measurement signal whose period is $\mathrm{L}$ are input. Then, the second period of the output signal, which is the result of the circular convolution, is extracted and subjected to the DFT to obtain $H(k) S(k)$. After this process, $H(k) S(k)$ is multiplied by the inverse characteristic $1 / S(k)$ of the measurement signal to obtain the frequency response $H(k)$ of the unknown system.

In an actual measurement the noise $N_{0}(k)$ is added to $H(k) S(k)$ as shown in Fig. 1(d). Thus, when $H(k) S(k)+$ $N_{0}(k)$ is multiplied by the inverse characteristic $1 / S(k)$, $H(k)+N_{0}(k) / S(k)$ is obtained where $N_{0}(k) / S(k)$ is the measurement error caused by noise (hereafter, the noise component). This indicates that the magnitude of the noise component depends on the spectrum of the measurement signal $S(k)$.

By applying the inverse DFT to $H(k)+N_{0}(k) / S(k)$ the impulse response $h(n)+n_{1}(n)$ is obtained where $n_{1}(n)$ represents the inverse DFT of $N_{0}(k) / S(k)$. Figs. 2(a)-2(c) show model diagrams of the impulse response measured in the time domain. Fig. 2(a) shows that the noise $n_{0}(n)$ is added to the impulse response measured using the impulse signal $\delta(n) . p_{N 0}$ is the power of $n_{0}(n)$.

Fig. 2(b) shows the model diagram of a measurement signal $s(n)$. In this figure $L$ is the signal length, i.e., the defined length of the measurement signal. $J$ is the effective length, i.e., the length over which an effective signal exists; outside this section, the power of the signal is almost zero. For an MLS signal $J=L . p_{S}$ is the power of the signal in an effective section.

Fig. 2(c) shows the model diagram of the measured impulse response obtained using the measurement signal. The noise component $n_{1}(n)$ with the power $p_{N 1}$ is added.

\section{NOISE REDUCTION PERFORMANCE (NRP) OF MEASUREMENT SIGNALS}

\subsection{Theoretical Examination}

The power of the noise component, $p_{N 1}$, when the measurement signal $s(n)$ is used is significantly lower than that 
(a)

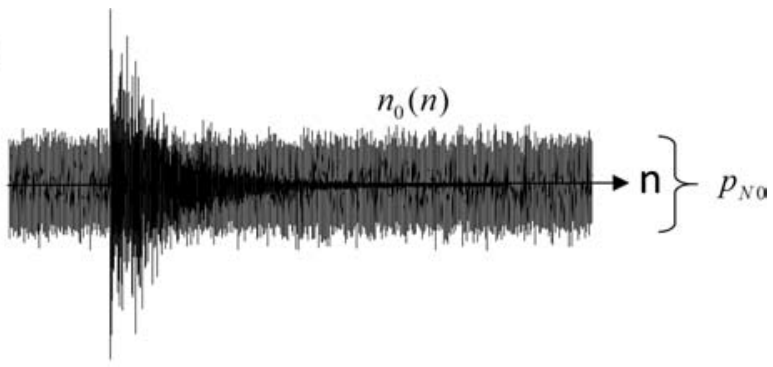

(b)

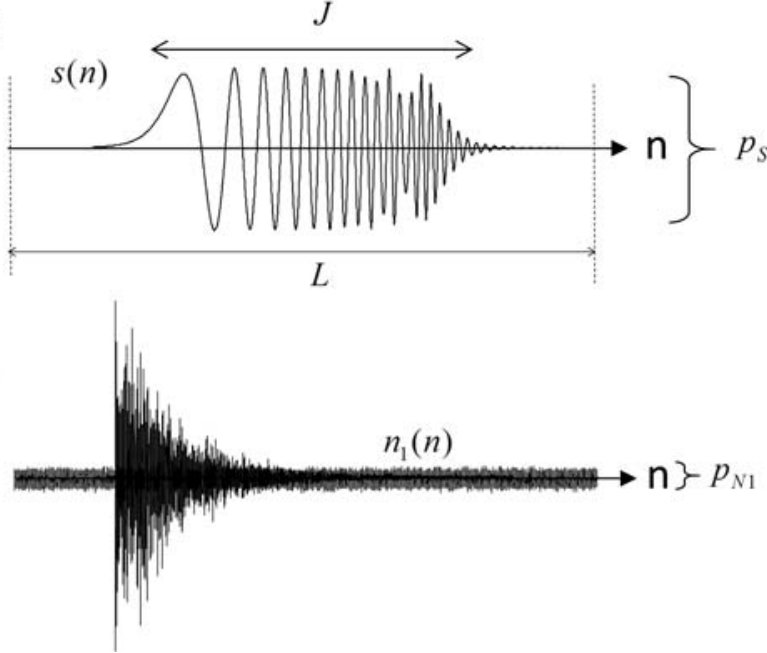

Fig. 2 Measurement result of impulse response and noise component. (a) Measurement result obtained using impulse signal $\delta(n)$ and noise component $n_{0}(n)$. (b) Model diagram of measurement signal $s(n)$. (c) Measurement result obtained using measurement signal $s(n)$ and noise component $n_{1}(n)$.

of the noise component, $p_{N 0}$, when the impulse signal $\delta(n)$ is used. Since the noise component $n_{1}(n)$ is obtained by applying the inverse DFT to $N_{0}(k) / S(k), p_{N 1}$ depends on the characteristics of the measurement signal spectrum $S(k)$.

In this study the noise reduction performance of measurement signals is evaluated using the $N R P$ defined by

$$
N R P=\frac{p_{N 0}}{p_{N 1}}
$$

Here, $p_{N i}(i=0,1)$ is the power of the noise component $n_{i}(n)$ defined by

$$
p_{N i}=E\left[\frac{1}{L} \sum_{n=0}^{L-1} n_{i}^{2}(n)\right],
$$

where $E[\cdot]$ is the expectation.

Parseval's relation, expressed by

$$
\sum_{n=0}^{L-1} n_{i}^{2}(n)=\frac{1}{L} \sum_{k=0}^{L-1}\left|N_{i}(k)\right|^{2},
$$

holds between $n_{i}(n)$ and $N_{i}(k)$, which is the L-point DFT of $n_{i}(n)$. From Eq. (3),

$$
E\left[\sum_{n=0}^{L-1} n_{i}^{2}(n)\right]=\frac{1}{L} \sum_{k=0}^{L-1} P_{N i}(k),
$$

where $P_{N i}(k)=E\left[\left|N_{i}(k)\right|^{2}\right]$ is the power spectrum of the noise component $n_{i}(n)$. The energy of the measurement signal, $E_{S}$, is given by

$$
E_{S}=\sum_{n=0}^{L-1} s^{2}(n)=\frac{1}{L} \sum_{k=0}^{L-1}|S(k)|^{2} .
$$

From Fig. 1(d), the power spectrum of the noise component $P_{N 1}(k)$ included in the measurement result is given by

$$
\begin{aligned}
P_{N 1}(k) & =E\left[\left|N_{1}(k)\right|^{2}\right]=E\left[\left|N_{0}(k)\right|^{2} /|S(k)|^{2}\right] \\
& =P_{N 0}(k) /|S(k)|^{2} .
\end{aligned}
$$

The power spectra $|\hat{S}(k)|^{2}$ and $\hat{P}_{N 0}(k)$ obtained by normalizing $|S(k)|^{2}$ and $P_{N 0}(k)$, respectively, using the respective mean values are defined by

$$
|\hat{S}(k)|^{2}=\frac{|S(k)|^{2}}{\frac{1}{L} \sum_{k=0}^{L-1}|S(k)|^{2}}
$$

and

$$
\hat{P}_{N 0}(k)=\frac{P_{N 0}(k)}{\frac{1}{L} \sum_{k=0}^{L-1} P_{N 0}(k)} .
$$

$|\hat{S}(k)|^{2}$ and $\hat{P}_{N 0}(k)$ have the following relationship.

$\sum_{k=0}^{L-1}|\hat{S}(k)|^{2}=\sum_{k=0}^{L-1} \hat{P}_{N 0}(k)=L$.

By solving Eqs. (7) and (8) for $|S(k)|^{2}$ and $P_{N 0}(k)$, respectively, we obtain

$|S(k)|^{2}=\left\{\frac{1}{L} \sum_{k=0}^{L-1}|S(k)|^{2}\right\} \cdot|\hat{S}(k)|^{2}$

and

$$
P_{N 0}(k)=\left\{\frac{1}{L} \sum_{k=0}^{L-1} P_{N 0}(k)\right\} \cdot \hat{P}_{N 0}(k) .
$$

On the right sides of Eqs. (10) and (11), the first component (for example, $1 / L \sum_{k=0}^{L-1}|S(k)|^{2}$ ) represents the signal energy and the second component represents a relative spectral shape independent of energy (for example, $|\hat{S}(k)|^{2}$ or $\left.\hat{P}_{N 0}(k)\right)$. 
Using Eqs. (2), (4), (5), (6), (9), (10), and (11), NRP in Eq. (1) can be represented as follows.

$$
\begin{aligned}
N R P & =\frac{p_{N 0}}{p_{N 1}}=\frac{E\left[\frac{1}{L} \sum_{n=0}^{L-1} n_{0}^{2}(n)\right]}{E\left[\frac{1}{L} \sum_{n=0}^{L-1} n_{1}^{2}(n)\right]} \\
& =\frac{\sum_{k=0}^{L-1} P_{N 0}(k)}{\frac{\sum_{k=0}^{L-1}}{L-1} P_{N 1}(k)}=\frac{\sum_{k=0}^{L-1} P_{N 0}(k)}{\sum_{k=0}^{L-1} \frac{P_{N 0}(k)}{|S(k)|^{2}}} \\
& =\frac{\sum_{k=0}^{L-1}\left\{\frac{1}{L} \sum_{k=0}^{L-1} P_{N 0}(k)\right\} \cdot \hat{P}_{N 0}(k)}{\left\{\sum_{k=0}^{L-1} \frac{\left\{\frac{1}{L} \sum_{k=0}^{L-1} P_{N 0}(k)\right\} \cdot \hat{P}_{N 0}(k)}{\left\{\frac{1}{L} \sum_{k=0}^{L-1}|S(k)|^{2}\right\} \cdot|\hat{S}(k)|^{2}}\right.} \\
= & \frac{1}{L} \sum_{k=0}^{L-1}|S(k)|^{2} \cdot \frac{\sum_{k=0}^{L-1} \hat{P}_{N 0}(k)}{\sum_{k=0}^{L-1}\left\{\hat{P}_{N 0}(k) /|\hat{S}(k)|^{2}\right\}} \\
= & E_{S} \cdot \frac{L}{\sum_{k=0}^{L-1}\left\{\hat{P}_{N 0}(k) /|\hat{S}(k)|^{2}\right\}} .
\end{aligned}
$$

The first component of the final term in Eq. (12) is the energy of the measurement signal, whereas the latter component of the final term is determined by the power spectral shapes of the noise $\hat{P}_{N 0}(k)$ and measurement signal $|\hat{S}(k)|^{2}$ and is independent of the measurement signal energy.

\subsection{Energy of Measurement Signal and NRP}

$N R P$ is proportional to the energy of the measurement signal $E_{S}$. The energy of the measurement signal is determined as $E_{S}=J \cdot p_{S}$ from Fig. 2(b). Thus, NRP increases when $p_{S}$ increases with increasing measurement signal amplitude or when the effective length $J$ increases.

Note that when the ratio of the effective length $J$ to the total signal length $L$ is kept constant, the SN ratio, i.e., the ratio of signal energy to noise energy in the received signal, remains constant even when $L$ increases (accordingly, $J$ increases). Also, $P_{N 1}(k)=P_{N 0}(k) /|S(k)|^{2}$ takes almost the same value at the normalized frequencies $k / L(k=0,1,2, \cdots, L / 2)$ regardless of the signal length $L$. However, the power of the noise component, $p_{N 1}$, obtained by dividing the noise energy by $L$, decreases with increasing $L$, while the impulse response waveform maintains the same height.

Also note that the second component of the final term in Eq. (12) is almost independent of $L$ because the denominator is almost proportional to $L$.

\subsection{Power Spectral Shape of Measurement Signal and NRP}

In this subsection the relationship between the power spectral shape of the measurement signal and NRP is examined.

Case 1: When the measurement signal $S(k)$ has a white spectrum such as a linearly SS signal or an MLS signal, the spectrum of $S(k)$ is given by

$$
|S(k)|^{2}=C_{1} . \quad C_{1}: \text { constant }
$$

By substituting Eq. (13) into Eq. (7), we obtain

$$
|\hat{S}(k)|^{2}=1
$$

for all $k$. By substituting Eq. (14) into Eq. (12), and using Eq. (9), we obtain

$$
N R P=E_{S} .
$$

Thus, the NRP of a measurement signal with a white spectrum is identical to the energy of the measurement signal. This NRP value does not depend on the noise spectrum.

Case 2: When the measurement signal $S(k)$ has a noisewhitening spectrum (NW signal), the spectrum is given by

$$
|S(k)|^{2}=C_{2} \cdot P_{N 0}(k) . \quad C_{2} \text { : constant }
$$

Using Eq. (16), the power spectrum of the noise component $P_{N 1}(k)$ is given by

$$
P_{N 1}(k)=\frac{P_{N 0}(k)}{|S(k)|^{2}}=\frac{P_{N 0}(k)}{C_{2} \cdot P_{N 0}(k)}=\frac{1}{C_{2}} .
$$

This is a white spectrum independent of the frequency $k$.

Substituting Eq. (16) into Eq. (7) and using Eq. (8) gives

$$
|\hat{S}(k)|^{2}=\frac{C_{2} \cdot P_{N 0}(k)}{\frac{1}{L} \sum_{k=0}^{L-1} C_{2} \cdot P_{N 0}(k)}=\hat{P}_{N 0}(k) .
$$

Then, by substituting Eq. (18) into Eq. (12), we obtain

$$
N R P=E_{S} .
$$

Comparing Eqs. (15) with (19), the NRP of the measurement signal with a noise-whitening spectrum is found to be the same as that of the measurement signal with a white spectrum.

Case 3: When the measurement signal $S(k)$ has an MN spectrum (MN signal), the spectrum is given by

$$
|S(k)|^{2}=C_{4} \cdot \sqrt{P_{N 0}(k)} . \quad C_{4} \text { : constant }
$$

The rationale behind Eq. (20) is given in Appendix 1.

By substituting Eq. (20) into Eq. (7) we obtain

$$
|\hat{S}(k)|^{2}=\frac{\sqrt{P_{N 0}(k)}}{\frac{1}{L} \sum_{k=0}^{L-1} \sqrt{P_{N 0}(k)}} .
$$

By substituting Eq. (21) into Eq. (12), based on the fact that $\sum_{k=0}^{L-1} \sqrt{P_{N 0}(k)}$ is not a function of $\mathrm{k}$ but a constant value, and using Eq. (8), we obtain

$$
\begin{aligned}
N R P & =E_{S} \cdot \frac{\frac{L}{\sum_{k=0}^{L-1}\left\{\hat{P}_{N 0}(k) \cdot\left(\frac{1 / L \cdot \sum_{k=0}^{L-1} \sqrt{P_{N 0}(k)}}{\sqrt{P_{N 0}(k)}}\right)\right\}}}{}=E_{S} \cdot \frac{L}{\frac{1}{L} \sum_{k=0}^{L-1} \sqrt{P_{N 0}(k)} \cdot \sum_{k=0}^{L-1} \frac{\hat{P}_{N 0}(k)}{\sqrt{P_{N 0}(k)}}} \\
& =E_{S} \cdot \frac{L^{2} \cdot \frac{1}{L} \sum_{k=0}^{L-1} P_{N 0}(k)}{\sum_{k=0}^{L-1} \sqrt{P_{N 0}(k)} \cdot \sum_{k=0}^{L-1} \frac{P_{N 0}(k)}{\sqrt{P_{N 0}(k)}}} \\
& =E_{S} \cdot \frac{L \cdot \sum_{k=0}^{L-1} P_{N 0}(k)}{\left(\sum_{k=0}^{L-1} \sqrt{P_{N 0}(k)}\right)^{2}} .
\end{aligned}
$$




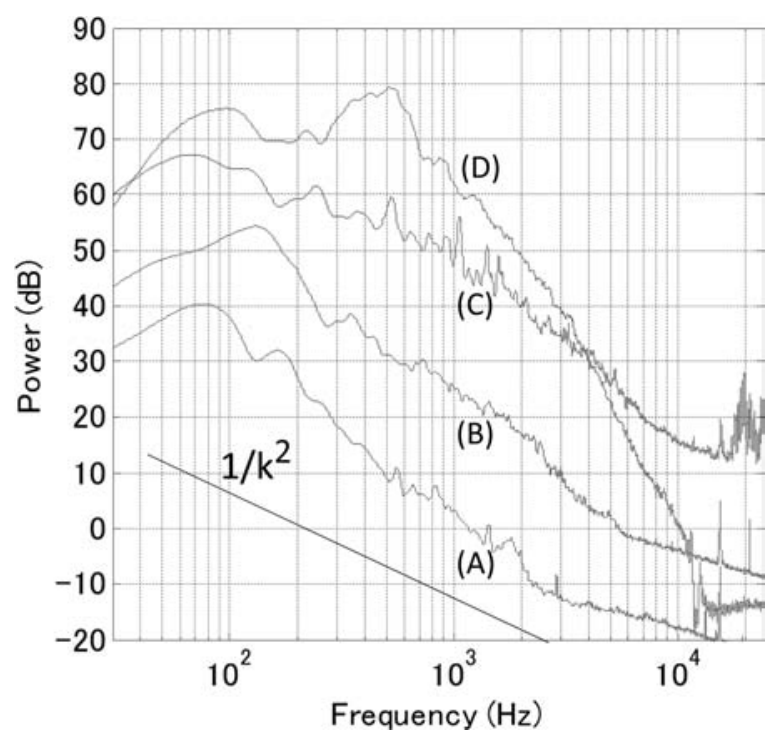

Fig. 3 Spectra of room noises used in simulation.

Here, the variance of the amplitude spectrum of the noise component $\sqrt{P_{N 0}(k)}(k=0,1,2, \cdots, L-1)$ given by

$$
\begin{aligned}
& \frac{1}{L} \sum_{k=0}^{L-1}\left(\sqrt{P_{N 0}(k)}\right)^{2}-\left(\frac{1}{L} \sum_{k=0}^{L-1} \sqrt{P_{N 0}(k)}\right)^{2} \\
= & \frac{1}{L^{2}}\left\{L \cdot \sum_{k=0}^{L-1} P_{N 0}(k)-\left(\sum_{k=0}^{L-1} \sqrt{P_{N 0}(k)}\right)^{2}\right\}
\end{aligned}
$$

is considered. The magnitude of the final term in Eq. (22) increases as the last term in Eq. (23), that is, the variance of $\sqrt{P_{N 0}(k)}$ increases. In other words, the higher the variance of the amplitude spectrum as a function of frequency, the higher the NRP obtained using the MN signal.

The inequality

$$
1 \leq \frac{L \cdot \sum_{k=0}^{L-1} P_{N 0}(k)}{\left(\sum_{k=0}^{L-1} \sqrt{P_{N 0}(k)}\right)^{2}} \leq L
$$

holds for the latter component of the final term in Eq. (22). Therefore, the achievable NRP of the MN signal depends on the shape of the noise spectrum and is theoretically at most $L$-fold higher than that of the signal with a white spectrum.

\section{SIMULATION OF NRP}

To verify the results of the theoretical examination in the previous section the impulse response measurement in a noisy environment was simulated.

\subsection{Conditions for Simulation}

In the simulation the measurement signal and the impulse response of a loudspeaker were convolved and then noise was added. Thus, a received sound signal was simulated. Fig. 3 shows the spectra of the four types of actual room noise used in the simulation. In the figure each spectrum is plotted with a different power level to make the spectral shape more understandable.
Table $1 N R P$ values obtained for four measurement signals.

\begin{tabular}{lllll}
\hline $\begin{array}{l}\text { (a) Measured NRP }(\mathrm{dB}) \\
\text { Signal Spectrum }\end{array}$ & Noise A & Noise B & Noise C & Noise D \\
\hline White & 39.9 & 39.6 & 40.5 & 40.6 \\
NW & 40.7 & 38.5 & 40.5 & 40.0 \\
Pink & 51.8 & 49.3 & 45.9 & 45.2 \\
MN & 58.2 & 55.7 & 51.7 & 54.0 \\
\hline
\end{tabular}

(b) NRP values $(\mathrm{dB})$ normalized by that of white signal for corresponding noise.

\begin{tabular}{lllll} 
Signal Spectrum & Noise A & Noise B & Noise C & Noise D \\
\hline White & 0 & 0 & 0 & 0 \\
NW & 0.8 & -1.1 & 0.0 & -0.6 \\
Pink & 16.5 & 14.3 & 10.0 & 9.2 \\
MN & 18.3 & 16.1 & 11.2 & 13.3 \\
\hline
\end{tabular}

All noises were recorded in our laboratory rooms that had an average volume of $100 \mathrm{~m}^{3}$. The main sources of noises A and B were air conditioners. In addition to an air conditioner there were several computers and home electrical appliances, such as a refrigerator, in the room in which noise $\mathrm{C}$ was recorded. Noise $\mathrm{D}$ was recorded in a room next to a room containing machinery, so its spectrum was high at frequencies of approximately $300-600 \mathrm{~Hz}$.

As measurement signals, four SS signals with one of the following spectra were used. Here, $C_{i}(i=1,2,3,4)$ is constant.

(a) White spectrum (e.g., linearly SS signal): $|S(k)|^{2}=$ $C_{1}$

(b) NW spectrum: $|S(k)|^{2}=C_{2} \cdot P_{N 0}(k)$

(c) Pink spectrum (e.g., log-SS signal): $|S(k)|^{2}=C_{3} / k$

(d) MN spectrum: $|S(k)|^{2}=C_{4} \cdot \sqrt{P_{N 0}(k)}$

The sampling frequency was $48 \mathrm{kHz}$. The target frequency range was $70 \mathrm{~Hz}-24 \mathrm{kHz}$, and a high-pass filter with a cutoff frequency of $70 \mathrm{~Hz}$ was used for the received sound signal. The amplitude $A$, signal length $L$, and effective length $J$ were set to $0.8,2^{16}$, and $2^{15}$, respectively, for all signals. This makes the energies $\left(E_{\mathrm{S}}\right)$ of the four measurement signals identical.

The noise level $p_{N 0}$ was set so that the $\mathrm{SN}$ ratio of the received signal was about $6 \mathrm{~dB}$, although $p_{N 0}$ does not affect $N R P$. Constants $\mathrm{C}_{\mathrm{i}}(\mathrm{i}=1,2,3,4)$ were not set explicitly because they were determined from the signal energy Es and noise power spectrum $P_{N 0}(k)$ and by using Eq. (5).

\subsection{Simulation Results}

Table 1(a) shows the $N R P(\mathrm{~dB})$ characteristics of the four measurement signals for the four types of noise (noises A, B, C, and D). As shown in Table 1(a), the NRP of the white signal is approximately $40 \mathrm{~dB}$ regardless of the type of noise. From Eq. (15), the NRP of the white signal is given by the value of $E_{\mathrm{S}}$ for the measurement signal regardless of the type of noise. $E_{\mathrm{S}}$ for the SS signal with amplitude $A$ and effective length $J$ is given by

$$
E_{S}=J \cdot A^{2} / 2 .
$$



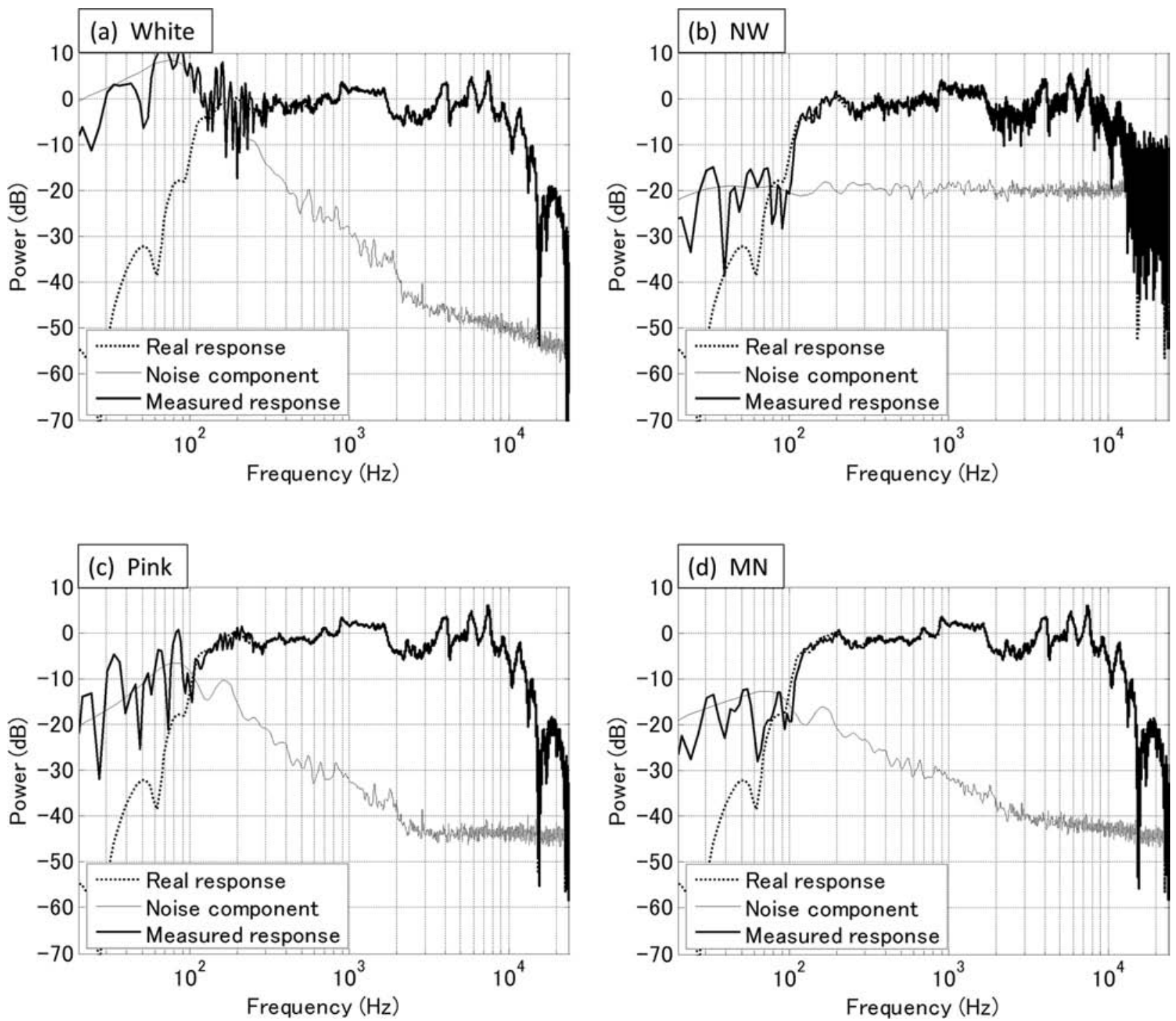

Fig. 4 Frequency responses of measurement results obtained using four measurement signals for noise A. (a) White signal, (b) NW signal, (c) pink signal, (d) MN signal.

By substituting the values of $J$ and $A$ given in the previous subsection into Eq. (25),

$$
E_{S}=10 \cdot \log _{10}\left(2^{15} \cdot(0.8)^{2} / 2\right)=40.3[\mathrm{~dB}]
$$

is obtained. This theoretical value is in good agreement with the simulation result.

Table 1(b) shows the results obtained by normalizing the $N R P$ in Table 1(a) using the NRP of the white signal for the corresponding noise. From Table 1(b), the $N R P$ of the NW signal was found to be almost equal to that of the white signal for the four types of noise. This finding is also in agreement with the theoretical result.

The NRP of the MN signal is the highest among the four measurement signals and is $11-18 \mathrm{~dB}$ higher than that of the white signal. NRP increases for a noise spectrum with a sharper peak and hence having a higher variance of the amplitude in the frequency axis, which also agrees with the theoretical result.

The NRP values of the pink signal for noises $\mathrm{A}, \mathrm{B}$, and $\mathrm{C}$ are similar to those of the $\mathrm{MN}$ signal. The difference in NRP between the pink and MN signals is small, i.e., approximately $1-2 \mathrm{~dB}$. This is because noises A, B, and C have a monotonically decreasing spectrum that is similar to the $1 / k^{2}$ (power) spectrum, and the $\mathrm{MN}$ spectrum for the $1 / k^{2}$ noise spectrum is a $1 / \mathrm{k}$ (pink) spectrum. However, the $N R P$ of the pink signal is lower for noise $\mathrm{D}$ because the spectrum of noise $\mathrm{D}$ deviates from the $1 / k^{2}$ spectrum, and $N R P$ is about $4 \mathrm{~dB}$ lower than that for the MN signal. To increase $N R P$ by $4 \mathrm{~dB}$, it is necessary to increase the length of the measurement signal 2.5 -fold.

Figs. 4(a)-4(d) show the real frequency responses, the spectra of the noise components, and the responses obtained using the four measurement signals for noise A. Fig. 4(a) shows the result obtained using the white signal. As shown in the figure, the spectrum of noise $\mathrm{A}$ is unchanged resulting in a low $\mathrm{SN}$ ratio in the low-frequency range.

Fig. 4(b) shows the result obtained using the NW signal. The noise is whitened and the noise level in the lowfrequency range is reduced. However, the noise level in the high-frequency range significantly increases. As a result, the noise component energy over the entire range is equivalent to that of the white signal. 

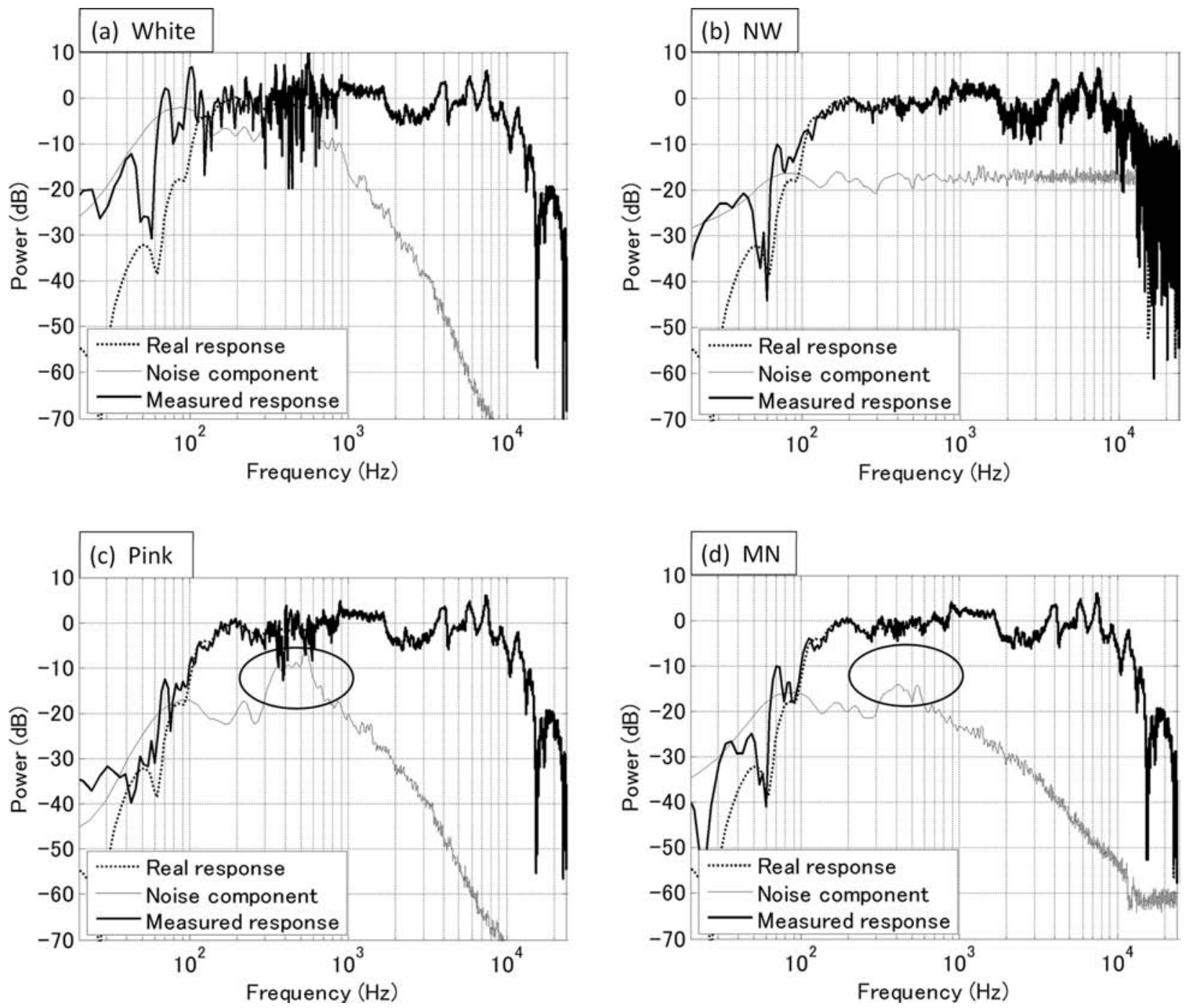

Fig. 5 Frequency responses of measurement results obtained using four measurement signals for noise C. (a) White signal, (b) NW signal, (c) pink signal, (d) MN signal.

Figs. 4(c) and 4(d) show the results obtained using the pink and MN signals, respectively. In both cases the noise level in the low-frequency range is reduced with an acceptable increase in the noise level in the high-frequency range. However, upon close observation, the noise component obtained using the pink signal is smaller than that obtained using the MN signal in the high-frequency range. In contrast, the noise component obtained using the pink signal is larger than that obtained using the MN signal in the low-frequency range. This causes the fluctuation in the frequency response obtained using the pink signal at frequencies of less than $300 \mathrm{~Hz}$. The results for noises B and $\mathrm{C}$ were similar to those for noise $\mathrm{A}$.

Figs. 5(a)-5(d) show the noise component spectra and measured frequency responses for noise D. The measured response using the white signal (Fig. 5(a)) fluctuates under the influence of a noise component in the low-frequency range, and the measured response using the NW signal (Fig. 5(b)) fluctuates under the influence of a noise component in the high-frequency range, similarly to Figs. 4(a) and $4(b)$.
Figs. 5(c) and 5(d) show the results obtained using the pink and MN signals, respectively. In both cases the noise level in the low-frequency range is reduced with an acceptable increase in the noise level in the high-frequency range. In the case of the pink signal (Fig. 4(c)), however, the noise component at approximately $500 \mathrm{~Hz}$ cannot be reduced. In contrast, in the case of the MN signal (Fig. 4(d)), the noise component at this frequency is more reduced than that in the case of the pink signal because the MN signal adapts to the actual noise spectrum. This explains the higher NRP of the MN signal than that of the pink signal, as shown in Table 1.

Figs. 6 and 7 show the waveforms of the noise components using the four measurement signals for noise A (Fig. 6) and noise D (Fig. 7). Figs. 6(a) and 7(a) show the waveforms of the noise components obtained using the white signal. Because the spectra of these noise components are the same as those of the added ambient noises, these waveforms are similar to those of the added ambient noises. Figs. 6(b) and 7(b) show the waveforms of the noise components obtained using the NW signal. These are 


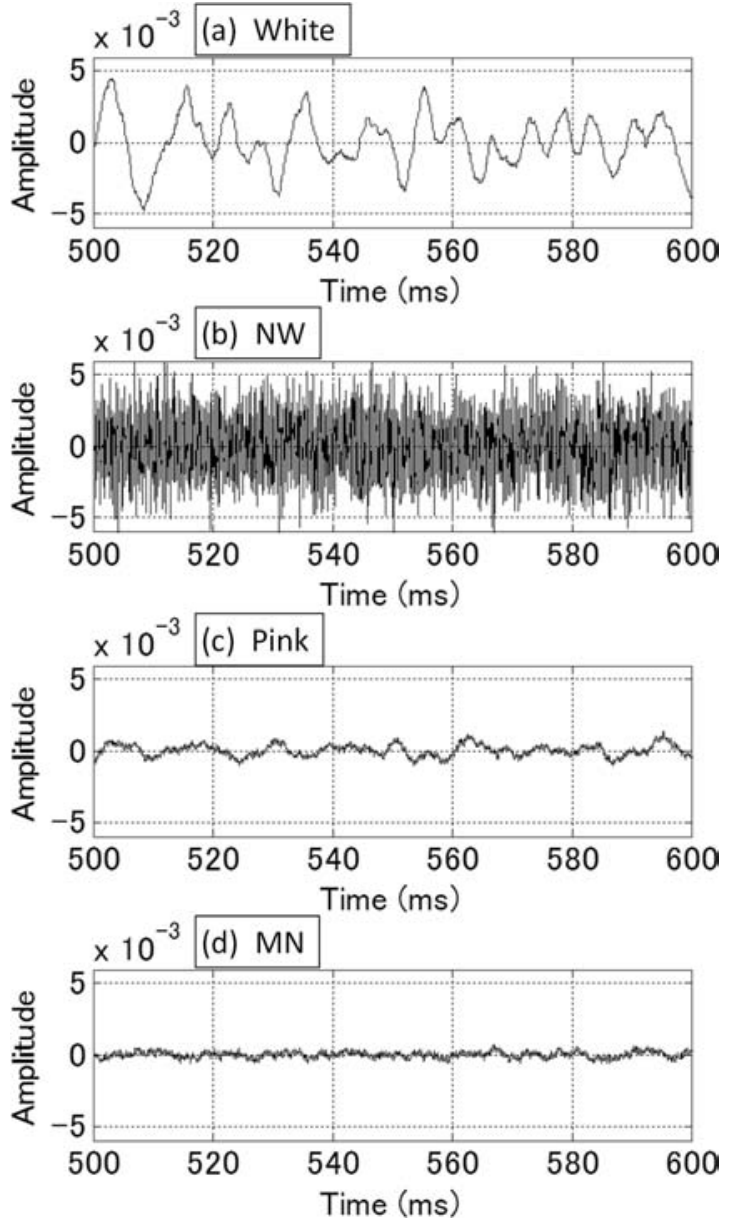

Fig. 6 Noise component waveforms obtained using four measurement signals for noise A. (a) White signal, (b) NW signal, (c) pink signal, (d) MN signal.

white-noise waveforms and their power levels are almost the same as those in Figs. 6(a) and 7(a).

We can see that the power levels of the noise component waveforms obtained using the pink signal (Figs. 6(c) and 7(c)) are smaller than those in Figs. 6(a), 6(b), 7(a), and 7(b), and the power levels of the noise component waveforms obtained using the MN signal (Figs. 6(d) and 7(d)) are even smaller. These results show good agreement with those in Table 1.

\section{CONCLUSIONS}

In this study the noise reduction performance in terms of $N R P$ (the amount of the reduced energy of the noise component included in the impulse response) obtained using various impulse response measurement signals was theoretically examined.

The measurement signals were swept sine or pseudonoise signals having four types of power spectrum. The power spectra were white, pink, noise-whitening (NW), and minimum noise $(\mathrm{MN})$. The NW power spectrum was the same as the power spectrum of the ambient noise, $P_{N 0}(k)(k$ : discrete frequency number), and the $\mathrm{MN}$ power spectrum was the square root of $P_{N 0}(k)$.

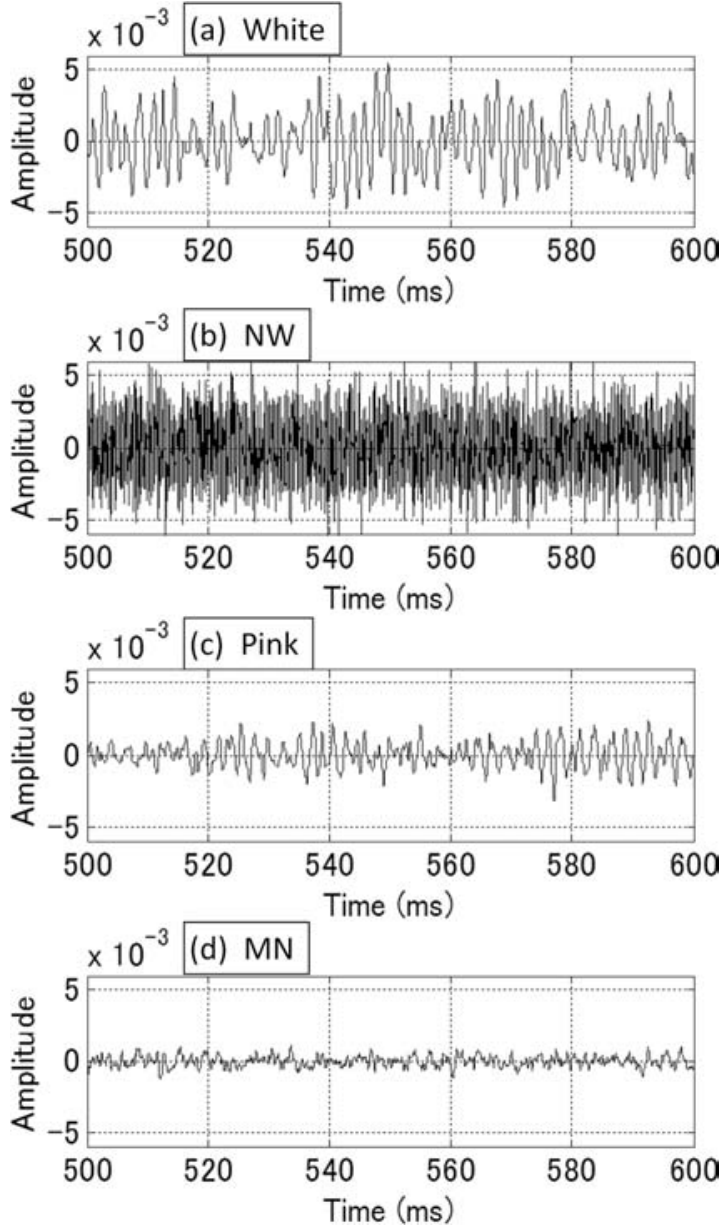

Fig. 7 Noise component waveforms obtained using four measurement signals for noise C. (a) White signal, (b) NW signal, (c) pink signal, (d) MN signal.

Equations that represent $N R P$ in terms of the measurement signal energy and the power spectral shapes of both the measurement signal and the ambient noise were derived. The following results were obtained from the theoretical equations and the results of the simulation carried out to verify the equations.

1) $N R P$ is proportional to the energy of the measurement signal $E_{\mathrm{S}}$.

2) The power spectral shape of the noise component included in the measurement results obtained using a white signal is identical to that of the added ambient noise. The obtained $N R P$ value does not depend on the noise spectrum.

3) The NW signal that whitens the noise component included in the measurement signal greatly reduces noise in the frequency range where an added noise has a large energy. However, the noise level increases in the frequency range where an added noise has a small energy. As a result, the NRP of the NW signal is equivalent to that of a white signal.

4) The MN signal has the highest $N R P$, which is $11-18$ $\mathrm{dB}$ higher than that of a white signal for the typical room noises used in the simulation. 
5) The measurement signal with a pink spectrum has a similar NRP to the MN signal for noise with a spectrum close to the $1 / k^{2}$ spectrum. However, as the noise spectrum deviates from the $1 / k^{2}$ spectrum, the difference in $N R P$ between the two signals increases. The maximum difference is $4 \mathrm{~dB}$ for the noise used in the simulation. This difference corresponds to 2.5-fold signal length.

Summarize the results; the overall noise reduction performance for white signals and NW signals is actually the same. Only the MN signal that can minimize the noise component showed a significant improvement in NRP. The pink spectrum measurement signal showed good NRP in the presence of $1 / \mathrm{k}^{2}$ spectrum noise, where $\mathrm{k}$ is the discrete frequency number, but worse performance with other types of noise. This supports the conclusion that using the MN signal that has a power spectrum that is the square root of the power spectrum of the noise is the best method of reducing the effect of noise on the measured impulse response.

\section{REFERENCES}

[1] H. Kuttruff, Room Acoustics (Elsevier Applied Science, Essex 1991).

[2] J. Blauert, Spatial Hearing (MIT Press, Cambridge 1983).

[3] Y. Huang and J. Benesty, Eds., Audio Signal Processing for Next-Generation Multimedia Communication Systems (Kluwer Academic Publisher, Norwell 2004).

[4] N. Aoshima, "Computer-Generated Pulse Signal Applied for Sound Measurement," J. Acoust. Soc. Am., vol. 69, no. 5, pp. 1484-1488 (May 1981).

[5] Y. Suzuki, F. Asano, H. Kim and T. Sone, "An Optimum Computer-Generated Pulse Signal Suitable for the Measurement of Very Long Impulse Responses," J. Acoust. Soc. Am., vol. 97, no. 2, pp. 1119-1123 (1995).

[6] S. Müller and P. Massarani, "Transfer-Function Measurement with Sweeps," J. Audio Eng. Soc., vol. 49, pp. 443-471 (2001 June).

[7] J. Borish, "An Efficient Algorithm for Measuring the Impulse Response Using Pseudorandom Noise," J. Audio Eng. Soc., vol. 31, pp. 478-488 (1983 Jul./Aug.).

[8] D. D. Rife and J. Vanderkooy, "Transfer-Function Measurement with Maximum-Length Sequences," J. Audio Eng. Soc., vol. 37, pp. 419-444 (1989 June).

[9] C. Dunn and M. O. Hawksford, "Distortion Immunity of MLS-Derived Impulse Response Measurements," $J$. Audio Eng. Soc., vol. 41, pp. 314-335 (1993 May).

[10] T. Fujimoto, "A Study of TSP Signal Getting Higher SN Ratio at Low Frequency Bands," Proc. Autumn Meet. Acoust. Soc. Jpn., pp. 433-434 (Sept. 1999) [in Japanese].

[11] A. Farina, "Simultaneous Measurement of Impulse Response and Distortion with a Swept-Sine Technique," presented at the 108th Convention of the Audio Engineering Society (2000 Feb.), convention paper 5093.

[12] G. B. Stan, J. J. Embrechts, and D. Archambeau, "Comparison of Different Impulse Response Measurement Techniques," J. Audio Eng. Soc., vol. 50, pp.249-262 (2002 Apr.).
[13] S. Weinzierl, A. Giese and A. Lindau, "Generalized Multiple Sweep Measurement," presented at the 126th Convention of the Audio Engineering Society (2009 May), convention paper 7767 .

[14] N. Moriya and Y. Kaneda, "Impulse Response Measurement that Maximizes Signal-to-Noise Ratio Against Ambient Noise," Acoust. Sci. \& Tech., vol. 28, no. 1, pp. 43-45 (Jan. 2007). Sample program can be found in http://www.asp.c.dendai.ac.jp/IR_mes_e01.html.

[15] N. Moriya and Y. Kaneda, "Optimum Signal for Impulse Response Measurement that Minimizes Error Caused by Ambient Noise," J. Acoust. Soc. Japan., vol. 64, no. 12, pp. 695-701 (Dec. 2008) [in Japanese].

\section{APPENDIX}

\section{SPECTRUM OF MEASUREMENT SIGNAL THAT CAN MINIMIZE THE NOISE COMPONENT [15]}

Assuming that the energy of the measurement signal $E_{\mathrm{S}}$ takes a value $\mathrm{E}_{\mathrm{SC}}$,

$$
E_{S}=\frac{1}{L} \cdot \sum_{k=0}^{L-1}|S(k)|^{2}=E_{S C},
$$

we obtain the power spectrum $|S(k)|^{2}$ of a measurement signal that can minimize the power of the noise component $p_{\mathrm{N} 1}$ included in the measurement result. Here, using Eqs. $(2),(4)$, and (6), $p_{\mathrm{N} 1}$ is given by

$$
\begin{aligned}
p_{N 1} & =E\left[\frac{1}{L} \sum_{n=0}^{L-1} n_{1}^{2}(n)\right] \\
& =\frac{1}{L^{2}} \sum_{k=0}^{L-1} P_{N 1}(k)=\frac{1}{L^{2}} \sum_{k=0}^{L-1} \frac{P_{N 0}(k)}{|S(k)|^{2}} .
\end{aligned}
$$

Using Lagrange's method of undetermined multipliers and ignoring the constant $1 / L^{2}$, the desired $|S(k)|^{2}$ can be derived as the solution of the next equation.

$$
\begin{aligned}
& \frac{\partial}{\partial|S(k)|^{2}}\left\{\sum_{k=0}^{L-1} \frac{P_{N 0}(k)}{|S(k)|^{2}}-\lambda\left(E_{S C}-\frac{1}{L} \cdot \sum_{k=0}^{L-1}|S(k)|^{2}\right)\right\} \\
& =-\frac{P_{N 0}(k)}{|S(k)|^{4}}+\frac{1}{L} \cdot \lambda=0 .
\end{aligned}
$$

From Eq. (A-3) we obtain

$$
|S(k)|^{2}=\sqrt{L} \cdot \frac{\sqrt{P_{N 0}(k)}}{\sqrt{\lambda}}
$$

By substituting Eq. (A-4) into Eq. (A-1) we obtain

$$
\frac{1}{\sqrt{L} \sqrt{\lambda}} \sum_{k=0}^{L-1} \sqrt{P_{N 0}(k)}=E_{S C} .
$$

From Eq. (A-5) we obtain

$$
\sqrt{\lambda}=\frac{1}{\sqrt{L} \cdot E_{S C}} \sum_{k=0}^{L-1} \sqrt{P_{N 0}(k)}
$$


By substituting Eq. (A-6) into Eq. (A-4) we obtain

$$
|S(k)|^{2}=L \cdot E_{S C} \cdot \frac{\sqrt{P_{N 0}(k)}}{\sum_{k=0}^{L-1} \sqrt{P_{N 0}(k)}} .
$$

By representing terms independent of the frequency $k$ in Eq. (A-7) as

$$
C_{4}=\frac{L \cdot E_{S C}}{\sum_{k=0}^{L-1} \sqrt{P_{N 0}(k)}},
$$

we obtain Eq. (20) in Subsec. 2.3.

\section{THE AUTHOR}

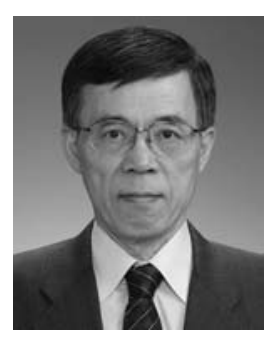

Yutaka Kaneda

Yutaka Kaneda was born in Osaka, Japan, in 1951. He received the B.E., M.E., and Doctor of Engineering degrees from Nagoya University, Nagoya, Japan, in 1975, 1977, and 1990. In 1977 he joined Nippon Telegraph and Telephone Corporation (NTT), Musashino, Tokyo, Japan, where he engaged in research on acoustic signal processing. In 2000 he joined Tokyo Denki University, Tokyo, Japan. He is currently a Professor of acoustic signal processing at the
Department of Information and Communication Engineering, Tokyo Denki University. His research interests include acoustical measurements, microphone array signal processing, and speech processing. Dr. Kaneda is a member of the Acoustical Society of Japan, the Acoustical Society of America, Audio Engineering Society, Institute of Electrical and Electronics Engineers, and the Institute of Electronics, Information and Communication Engineers of Japan. 